\title{
Evaluación de parámetros seminales de jóvenes universitarios de la ciudad de Lima - Perú
}

\section{Evaluation of Semen Quality in Young University Students from Lima - Perú}

\author{
Martin Daniel Arbaiza Barnechea ${ }^{1}$ \\ ${ }^{1}$ Universidad Nacional Agraria la Molina, Lima, Perú \\ Urol Colomb 2018;27:174-180.
}

\begin{abstract}
Address for correspondence Martin Daniel Arbaiza Barnechea, Lic. Blgo., Calle Manet $182 \mathrm{dpt} 102$ La calera de la merced surquillo, Lima, Perú (e-mail: arbaiza.b@hotmail.com).
\end{abstract}

\section{Resumen}

Palabras Clave

- Infertilidad

- Espermogramas

- Espermatozoides
Introducción A nivel mundial se está dando un fenómeno que cada vez es más común, la infertilidad.

Objetivo Evaluar las características seminales en jóvenes universitarios mediante espermatogramas utilizando el sistema C.A.S.A (Computer Assisted Sperm Analyzer, ISAS v1.2)

Materiales y métodos Se analizaron 30 muestras seminales de jóvenes universitarios entre 18 a 30 años de edad de la ciudad de Lima, Perú, que donaron hasta en tres distintas oportunidades y se comparó con los límites de referencia reportados por la Organización Mundial de Salud (OMS) y la Sociedad Europea de Reproducción Humana y Embriología (ESHRE). Se calcularon los estadísticos descriptivos, frecuencias y coeficientes de variación para todos los parámetros seminales, con un nivel de confianza de un $95 \%$ en el software SPSS v. 21

Resultados La gran mayoría de los valores evaluados cumplen con los criterios de la OMS Y ESHRE a excepción de la morfología 76,7\%. Esas alteraciones estructurales comprendían la cabeza y la pieza intermedia de los espermatozoides, las principales áreas afectadas fueron la longitud, el ancho, el área, la elipticidad, la elongación de la cabeza y el ancho de la pieza intermedia.

Conclusión No se encontraron asociaciones significativamente estadísticas entre los hábitos y los distintos parámetros seminales evaluados y se reveló que los valores encontrados superan los estándares recomendados por la OMS y ESHRE a excepción de la Morfología.

Introduction A worldwide phenomenon is becoming more and more common, infertility

Objective: Evaluate the seminal characteristics in young students by Spermograms and use the C.A.S.A system (Computer Assisted Sperm Analyzer, ISAS v1.2)

Materials and Methods 30 seminal samples of young university students between 18 and 30 years of age from the city of Lima, Peru, who donated up to three different opportunities and comparing with the reference limits reported by the World Health received

August 17, 2017

accepted

September 20, 2017

published online

March 29, 2018
DOI https://doi.org/ $10.1055 / \mathrm{s}-0038-1639540$ ISSN 0120-789X. eISSN 2027-0119.
Copyright ( $)$ 2018, Sociedad Colombiana License terms de Urología. Publicado por Thieme Revinter Publicações Ltda., Rio de Janeiro, Brazil. Todos los derechos reservados. 
Organization (WHO) and the European Society of Human Reproduction and Embryology (ESHRE). Descriptive statistics, frequencies and coefficients of variation were calculated for all seminal parameters. all analyzes were performed with a $95 \%$ confidence level in the SPSS v.21 software.

Results We determined that the most sperm values evaluated met WHO and ESHRE criteria with the exception of the morphology criteria $76.7 \%$. These structural alterations comprised the head and the intermediate part of the spermatozoa, the main areas affected were the length, width, area, ellipticity, elongation of the head and the width of the intermediate piece.

Conclusion There were no statistically significant associations between the habits and the different seminal parameters evaluated and it was revealed that the values found exceed the standards recommended by the WHO and ESHRE, except for Morphology

\section{Introducción}

A nivel mundial se está dando un fenómeno que cada vez es más común, la infertilidad. ${ }^{1-3}$ En el trascurso de los años la postergación del embarazo, ya sea por factores socioeconómicos, cambios en la conducta reproductiva, mayor expectativa de vida, desarrollo laboral, profesional, anticonceptivos y el mejoramiento de las técnicas de reproducción asistida, han desencadenado un aumento progresivo en la edad en la que mujeres y hombres logran el primer embarazo. ${ }^{3-5}$

En la actualidad, la edad es considerada un factor determinante en la calidad seminal, existe una relación directa entre la edad y el aumento de daño en el ADN espermático, ${ }^{2}$ lo que conlleva a una disminución en la concentración y la motilidad espermática. ${ }^{6-9}$

La Organización Mundial de la Salud considera que existe infertilidad masculina cuando hay alteración del espermatograma fundamentalmente en la calidad seminal, definida por la concentración de espermatozoides, la motilidad y morfología, asociada a alteraciones propias del líquido seminal. El espermatograma es una herramienta estándar para valorar la infertilidad masculina a través del análisis del líquido seminal, un buen estudio aporta una base para integrar una investigación complementaria y llegar a un diagnostico etiológico preciso, que permitirá tratar a los pacientes exitosamente. En la actualidad, estudios epidemiológicos determinan que las causas de esterilidad compartida de forma exclusiva obedecen a las alteraciones en el factor masculino en más del $50 \%$ de los casos. ${ }^{8,10}$

Los problemas en el área de reproducción humana tienden a ser diferentes en cada país, ya sea por factores ambientales, calidad de vida, tecnología, entre otros. De manera similar, los estudios de poblaciones sobre ese tema varían según el área estudiada. ${ }^{7,11-15}$

Los resultados seminales que se obtienen son muy difíciles de extrapolar a otra población, debido a que generalmente los parámetros seminales y la tasa de fertilidad son evaluados en muestras homogéneas de individuos (individuos que van a ser vasectomizados, donantes regulares de semen o individuos sanos con fertilidad probada), los cuales no necesariamente reflejan el estado reproductivo de la población en general. Adicionalmente, esos estudios no tienen en cuenta limitaciones relacionados con la población como abstinencia sexual, factores ambientales, estilo de vida y la metodología usada para el análisis seminal. ${ }^{12,15}$

Los estudios sobre parámetros seminales en la poblaciones y su variación relacionada con la geografía, han sido realizados principalmente en el hemisferio norte, ${ }^{4}$ muy poca información existe sobre ese tema en la poblaciones del trópico. ${ }^{9}$

En el transcurso de los años, se ha logrado mejorar los sistemas de evaluación seminal para de esa manera, eliminar la subjetividad inherente a la técnica, ya que la evaluación microscópica de la calidad del semen depende de la persona que la realice. ${ }^{16-18}$

El sistema computarizado de análisis de semen C.A.S.A (Computer Assisted Sperm Analyzer, ISAS v1.2) ha sido usado para reducir la variabilidad lo que se correlaciona significativamente con la fertilidad. Con el objetivo de solventar el problema de la subjetividad y de la variabilidad de algunos de los parámetros convencionales del espermograma clásico se empezó a utilizar ese sistema, el cual permite valorar de forma rápida, precisa y fiable tanto las variables cinéticas ${ }^{19}$ como las morfométricas. ${ }^{20,21}$

La importancia de este trabajo es que permite establecer una base de medidas estadísticas actuales de los parámetros seminales evaluados, para realizar comparaciones estadísticas que contribuyan a ampliar la perspectiva de las investigaciones en Perú, ya que actualmente no existen datos de análisis seminal con muestras variadas y al azar, teniendo en cuenta factores específicos como la metodología, abstinencia sexual, toma de muestra y evaluación morfológica mediante el sistema computarizado de análisis seminal C.A.S.A (Computer Assisted Sperm Analyzer, ISAS versus), en jóvenes universitarios peruanos.

\section{Métodos}

\section{Población de estudio}

Se evaluaron muestras seminales de 30 jóvenes universitarios que asistieron de manera voluntaria a las 
instalaciones del Banco Nacional de Semen, y donaron en tres distintas oportunidades.

Un total de 90 muestras fueron analizadas siguiendo el esquema indicado por la OMS 2010. ${ }^{22}$

Todos los participantes del estudio fueron informados adecuadamente mediante el consentimiento informado el cual accedieron a firmar y completaron un cuestionario epidemiológico clínico.

\section{Recolección de las muestras}

Las muestras seminales se recolectaron mediante masturbación después de 3 días de abstinencia sexual y fueron llevadas inmediatamente al laboratorio para iniciar su análisis.

\section{Análisis de las muestras}

Se realizó el análisis macroscópico y microscópico de las muestras según el manual para análisis seminal de la OMS $(2010) .^{22}$

En primer lugar, se evaluó la licuefacción, la viscosidad, el $\mathrm{pH}$ y el volumen seminal.

La movilidad se determinó observando al microscopio $10 \mu \mathrm{L}$ de la muestra de semen con el objetivo de 40X clasificando los espermatozoides en tres tipos de movilidad: tipo I aquellos con movimiento progresivo, tipo II con movimiento no progresivos y tipo III los espermatozoides inmóviles.

Para la viabilidad se mezclaron $10 \mu \mathrm{L}$ de semen y $10 \mu \mathrm{L}$ de eosina-Y y se contaron como viables los espermatozoides que excluían el colorante al observarlos al microscopio con el objetivo de 40X.

La concentración espermática se determinó mediante la cámara de Neubauer.

La morfología se evaluó mediante la tinción de Papanicolaou modificada para espermatozoides humanos.

Se tomaron en cuenta los parámetros de cabeza y pieza media espermática utilizando el sistema computarizado CASA (Computer Assisted Sperm Analyzer ISAS vs 1.2), el cual fue programado con los estándares para la evaluación morfológica en espermatozoides humanos (- Fig. 1). ${ }^{22,23}$

\section{Análisis estadístico}

Se calcularon los estadísticos descriptivos y frecuencias para todos los parámetros seminales considerados en el presente estudio. El coeficiente de variación se calculó dividiendo la desviación estándar con el promedio y se expresó en porcentaje. Todos los análisis se realizaron con un 95\% de confianza en el software SPSS v.21 para Windows.

\section{Resultados}

El rango de edad constituye un grupo heterogéneo entre 18 y 26 años con una media de $23 \pm 2,3$.

De los parámetros evaluados, solo los criterios de $\mathrm{pH}$, volumen, vitalidad, motilidad, concentración y recuento total espermático, cumplieron con los valores establecidos por la Organización Mundial de Salud y la Sociedad Europea de Reproducción Humana y Embriología (ESHRE), (- Tabla 1).

En cambio, el 76,7\% de las muestras seminales evaluadas, no cumple con los criterios mínimos de morfología espermática (-Tabla 2 ).

Se observó que existían alteraciones morfológicas en la cabeza y la pieza intermedia de los espermatozoides, y comparándolo con los valores considerados normales por la OMS se obtuvo que las principales áreas afectadas fueron la longitud, el ancho, el área, la elipticidad y la elongación de la cabeza al igual que el ancho de la pieza intermedia. Al compararlo también con los valores de la ESHRE, se obtuvo que las principales áreas afectadas fueron el ancho, el área y la elipticidad de la cabeza (-Tabla 2).

\section{Discusión}

De la literatura revisada, este es el primer estudio que evalúa los parámetros seminales en base a los espermogramas y al análisis morfológico evaluado en el equipo C.A.S.A (Computer

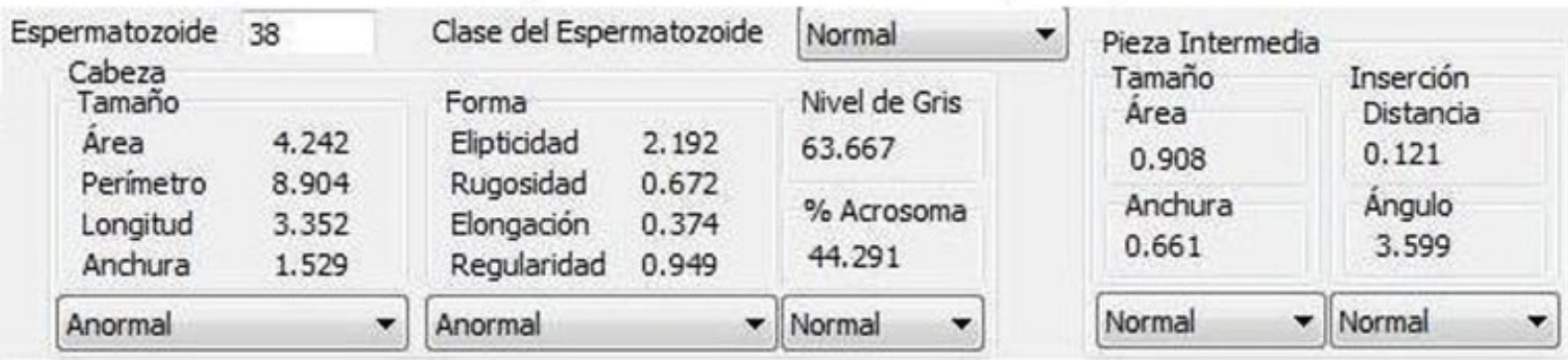

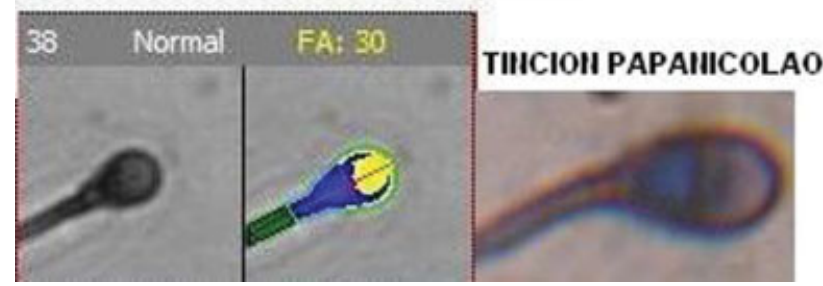

Fig. 1 Parámetros Sistema Computarizado de Análisis Seminal (C.A.S.A) ISAS vs 1.2. 


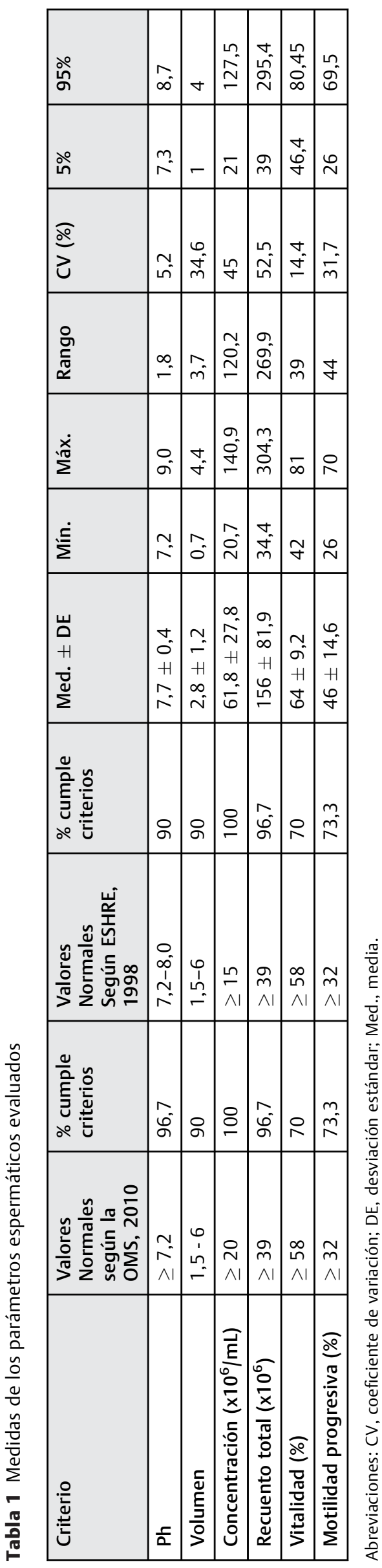

- Assisted Sperm Analyzer ISAS versus1.2) de la población joven de Lima -Perú.

En el presente estudio se reveló que los valores encontrados superan los estándares recomendados por la OMS $2010(\mathrm{pH}$, volumen, concentración, recuento total, vitalidad y Motilidad). Solo el parámetro de morfología espermática se encontró alterado en comparación con la OMS. Se determinó un $76,7 \%$ de incumplimiento con una media de $11,6 \pm 3,3$, con un mínimo de 7,5 y un máximo de 20 en las evaluaciones seminales.

Alrededor del mundo, ese parámetro también ha sido encontrado por debajo de lo considerado normal, Crazzolara y col. ${ }^{13}$ al evaluar una población Suiza de 34 individuos con una media de 9 , y un rango de variación entre $1-17$.

De igual forma Navarro y col. 2010, analizó 109 sujetos de una población Chile - Arica, encontrando una media de $15+/-7.9$, con un mínimo y máximo entre $0-43,1$, y Henao y col. 2013 evaluó una población de Colombia de 30 individuos, encontrando una media de 11.2, con un mínimo y máximo de 5-22.5.

Comparando los valores obtenidos con los demás autores, podemos determinar que evidentemente existe una disminución de los parámetros morfológicos creciente a lo largo de los años, mediante la evaluación clásica, pero esa estimación no nos permite determinar los distintitos parámetros que involucra una evaluación completa utilizando los sistemas computarizados de análisis seminal (C.A.S.A).

Las medidas de la anatomía morfológica espermática (-Tabla 2) nos dan una perspectiva distinta y abre la posibilidad a seguir investigando sobre las posibles explicaciones de por qué encontramos esas alteraciones.

Algunos autores estiman que la motilidad y la concentración son mejores predictores de fertilidad que la morfología espermática Natella y col. ${ }^{24}$ mientras que otros dan mayor importancia a la morfología o a la cinética de desplazamiento espermatozoide. Munuce y col. ${ }^{25}$ En nuestro caso, encontramos que la morfología espermática fue el único parámetro espermático que presentó alguna alteración en comparación con los parámetros cinéticos evaluados.

Brazil y col. ${ }^{1}$ nos indica en su investigación, que otra razón que podría explicar esa variación, sería las diferencias que pueden existir entre laboratorios referente a la subjetividad y variabilidad interpersonal al momento de evaluar las muestras seminales, lo que también fue reportado por Fisch, ${ }^{12}$ cada laboratorio trabaja con distintos tipos de metodología y en todo eso influyen estrechamente los factores ambientales y los hábitos de alimentación y tipo de vida en cada país.

Los valores obtenidos de Longitud de cabeza, anchura, área, perímetro, rugosidad, anchura de la pieza intermedia, y área son menores a los reportados en los trabajos de investigación realizados en distintas poblaciones. Aulesa y col. ${ }^{26}$ Maree y col. ${ }^{21}$ Bellastella y col. ${ }^{27}$

Una posible explicación la da Katz y col. ${ }^{3}$ en su estudio confirmando que las medidas de la cabeza de los espermatozoides disminuyen por el estrés ocasionado 


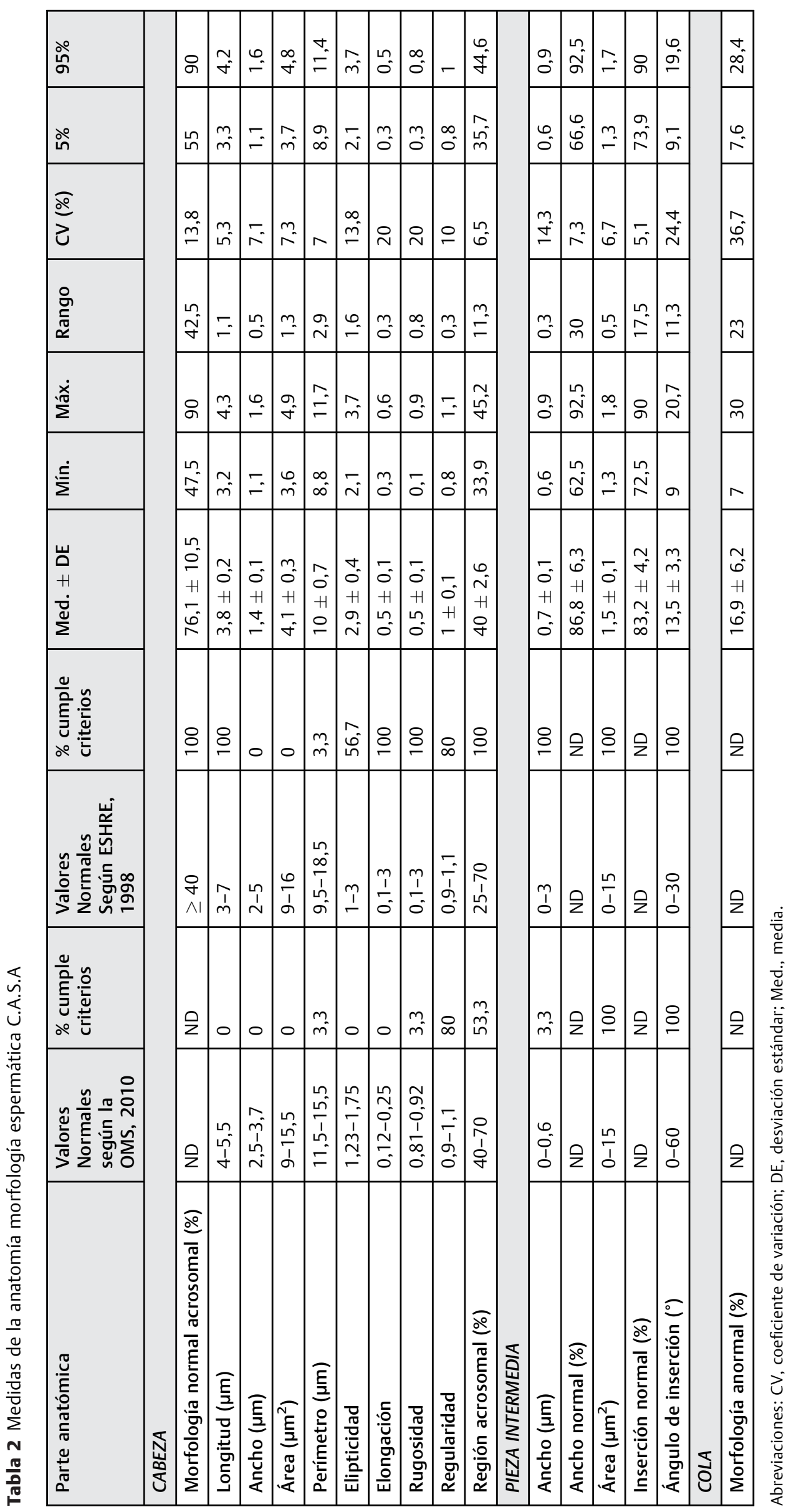


durante el proceso de tinción, específicamente durante la dispersión del colorante y el secado al aire, eso produce el hinchamiento de las células inmaduras de la cabeza, la perdida de gotas citoplasmáticas y la reducción del tamaño de la cabeza lo cual luego fue corroborado por Bellastella y col. ${ }^{27}$ Otro factor que podría influir, es la tinción utilizada para evaluar morfológicamente a los espermatozoide según Maree y col. ${ }^{21}$ que comparó 3 tinciones Papanicolaou, Rapidiff y SpermBlue, la longitud de cabeza, anchura, área, perímetro, elipticidad, elongación, rugosidad, región acrosomal, anchura de la pieza intermedia, área y ángulo de inserción que tienden a aumentar o disminuir en comparación con el semen fresco dependiendo de la tinción utilizada. Lo cual luego fue corroborado por qué Aksoy y col. ${ }^{20}$ A diferencia de los valores de elipticidad y elongación que se encuentran aumentado en comparación con los reportes de Maree y col. ${ }^{21}$

Los valores de región acrosomal son similares a los reportados por Aulesa y col. ${ }^{26}$ Maree y col. ${ }^{21}$ Bellastella y col. ${ }^{27}$ y el ángulo de inserción en comparación con los valores de Aulesa y col. ${ }^{26}$ son similares.

\section{Conclusiones}

De los resultados obtenidos podemos concluir que:

El 90\% de las muestras seminales analizadas cumplen con los criterios exigidos por la OMS $(2010)^{22}$ referente a los valores de $\mathrm{pH}$, concentración y recuento total. Yel $70 \%$ con los criterios de vitalidad, motilidad progresiva.

El 78\% de las muestras seminales analizadas no cumplen con el criterio de morfología normal, Esas alteraciones estructurales comprendían la cabeza y la pieza intermedia de los espermatozoides para el caso de los criterios exigidos por ESHRE (1998) ${ }^{28}$ y la OMS (2010). ${ }^{22}$

\section{Colaboradores}

Al Ingeniero Mg. Sc. Próspero Celestino Cabrera Villanueva, mi reconocimiento por su participación en este trabajo de investigación, por haber estado involucrado en el desarrollo de la concepción y el diseño del estudio, con sus consejos, recomendaciones y revisiones constantes, y la adquisición del respaldo financiero para el desarrollo experimental en el laboratorio del Banco Nacional de Semen de la Universidad Nacional Agraria La Molina.

\section{Agradecimientos}

Al Laboratorio de Reproducción Animal del Banco Nacional de Semen de la Universidad Nacional Agraria la Molina, Lima, Perú, por el uso de sus instalaciones para el desarrollo del trabajo de investigación Av. Raul Ferrero s/n, La Molina, Lima - Peru Email: bns@lamolina.edu.pe Los autores del manuscrito de referencia, declaran que no existen potenciales conflictos de interés.

Protección de personas y animales

Los autores declaran que los procedimientos seguidos se conformaron a las normas éticas del comité de experimentación humana responsable y de acuerdo con la Asociación Médica Mundial y la Declaración de Helsinki.

\section{Confidencialidad de los datos}

Los autores declaran que han seguido los protocolos de su centro de trabajo sobre la publicación de datos de pacientes.

Derecho a la privacidad y consentimiento informado Los autores han obtenido el consentimiento informado de los pacientes y/o sujetos referidos en el artículo. Este documento obra en poder del autor de correspondencia.

\section{Bibliografía}

1 Brazil C, Swan SH, Drobnis EZ, et al; Study for Future Families Research Group. Standardized methods for semen evaluation in a multicenter research study. J Androl 2004;25(04):635644

2 Flores R, Rodríguez C, Mallok A, Martínez G. Relación entre indicadores clínicos del espermograma y variables redox en infertilidad masculina. Rev Cub Farm 2011;25(03):361-379

3 Katz DF, Overstreet JW, Samuels SJ, Niswander PW, Bloom TD, Lewis EL. Morphometric analysis of spermatozoa in the assessment of human male fertility. J Androl 1986;7(04):203-210

4 Auger J, Kunstmann JM, Czyglik F, Jouannet P. Decline in semen quality among fertile men in Paris during the past 20 years. $\mathrm{N}$ Engl J Med 1995;332(05):281-285

5 Chávez J, Yarlequé J, Avalos E. Relación entre calidad del semen y la edad. Med Hered 2012;23(03):183-187

6 Avalos E, Barriento R, Chávez J, García A, Yarleque J. Relación entre la calidad del semen y la edad. Med Hered 2012;23(03): 183-187

7 Ivorra JA, García VP, Gasset RM, García JS, Díaz AF, Zamora JA. Relación entre la edad del varón y la calidad del estudio seminal. Experiencia en el área sanitaria 14 de la agencia valenciana de la salud. Arch Esp Urol 2008;61(06):705-710

8 Horta F, Madariaga M, García A, Hartel S, Smith R. Aumento del daño en el DNA espermático en varones mayores de 40 años. Med 2011;139(03):306-312

9 SerranoT. Una visión actual de la infertilidad masculina. Med-Rep 2011;4(03):103-109

10 Teppa-Garrán AD, Palacios-Torres A. Evaluación actual de la infertilidad masculina. Invest Clin 2004;45(04):355-370

11 Berdugo J, Andrade-Rocha F, Cardona-Maya W. Parámetros seminales en hombre fértiles de dos poblaciones suramericanas. Arch Esp Urol 2009;62(08):646-650

12 Fisch H, Braun SR. Trends in global semen parameter values. Asian J Androl 2013;15(02):169-173

13 Crazzolara S, Wunder D, Nägeli E, Bodmer C, Graf S, Birkhäuser MH. Semen parameters in a fertile Swiss population. Swiss Med Wkly 2007;137(11-12):166-172

14 Henao S, Cardona M. Evaluación de los parámetros seminales en 30 hombres con fertilidad probada y breve revisión de la literatura. Obstet Ginecol (Bucur) 2013;39(04):368-382

15 Navarro E, Cortés A, Monreal J, Ferreccio C. Análisis de las variables del espermograma en jóvenes sanos en Arica-Chile. Med 2010;138(12):1510-1516

16 Vásquez D, Vásquez F. Espermograma y su utilidad clínica, salud Uni norte 2007;23(02):220-230

17 Montoya A. Espermatogramas, Medicina y Laboratorio, ed. Médica Colombiana S.A. 2009; 15(3-4):145-169

18 Navarro E, Sarabia L. Evaluación y Estandarización de la Calidad del Espermograma: Nuevos Límites inferiores de Referencia. Int J Morphol 2011;29(03):885-890 
19 Krause W, Viethen G. Quality assessment of computer-assisted semen analysis (CASA) in the andrology laboratory. Andrologia 1999;31(03):125-129

20 Aksoy E, Murad T, Duman S, Cuce G. Assessment of Spermatozoa Morphology under Light Microscopy with Different Histologic Stains and Comparison of Morphometric Measurements. Int J Morphol 2012;30(04):1544-1550

21 Maree L, du Plessis SS, Menkveld R, van der Horst G. Morphometric dimensions of the human sperm head depend on the staining method used. Hum Reprod 2010;25(06):1369-1382

22 World Health Organization. "WHO Laboratory Manual for the examination and processing of human semen" Cambridge: Cambridge University. Fifth Edition; 2010:1-287

23 Dohle GR, Diemer T, Giwercman A, Jungwirth A, Kopa Z, Krausz C. Guía clínica sobre la infertilidad masculina, European Association of Urology. 2010: 1-69
24 Nallella KP, Sharma RK, Aziz N, Agarwal A. Significance of sperm characteristics in the evaluation of male infertility. Fertil Steril 2006;85(03):629-634

25 Munuce MJ, Cardona-Maya W, Berta CL. ¿Existe asociación entre la morfología normal del espermatozoide y su cinética de desplazamiento? Actas Urol Esp 2006;30(06):591-597

26 Aulesa C, Cabrera M, Benítez MY, Martínez M. Evaluación del sistema automatizado Sperm Class Analyzer (SCA) para análisis del semen. Lab Clin 2012;5(02):75-80

27 Bellastella G, Cooper TG, Battaglia M, et al. Dimensions of human ejaculated spermatozoa in Papanicolaou-stained seminal and swim-up smears obtained from the Integrated Semen Analysis System (ISAS(®)). Asian J Androl 2010;12(06):871-879

28 European Society of Human Reproduction and Embryology (ESHRE). Monographs. Manual on Basic Semen Analysis. Ed. U. Kvist and L. Bjorndahl. Oxford University Press; 2002 